\title{
miR-21 is associated with fibrosis and right ventricular failure
}

\author{
Sushma Reddy, ${ }^{1}$ Dong-Qing Hu, ${ }^{1}$ Mingming Zhao, ${ }^{1}$ Eddie Blay Jr., ${ }^{2}$ Nefthi Sandeep, ${ }^{1}$ Sang-Ging Ong, ${ }^{3}$ \\ Gwanghyun Jung, ${ }^{1}$ Kristina B. Kooiker, ${ }^{1}$ Michael Coronado, ${ }^{1}$ Giovanni Fajardo, ${ }^{1}$ and Daniel Bernstein ${ }^{1}$ \\ 'Department of Pediatrics, Division of Cardiology, Stanford University, Stanford, California, USA. ${ }^{2}$ Department \\ of Surgery, Temple University, Philadelphia, Pennsylvania, USA. ${ }^{3}$ Cardiovascular Institute, Stanford University, \\ Stanford, California, USA.
}

\begin{abstract}
Combined pulmonary insufficiency (PI) and stenosis (PS) is a common long-term sequela after repair of many forms of congenital heart disease, causing progressive right ventricular (RV) dilation and failure. Little is known of the mechanisms underlying this combination of preload and afterload stressors. We developed a murine model of PI and PS (PI+PS) to identify clinically relevant pathways and biomarkers of disease progression. Diastolic dysfunction was induced (restrictive RV filling, elevated RV end-diastolic pressures) at 1 month after generation of PI+PS and progressed to systolic dysfunction (decreased RV shortening) by 3 months. RV fibrosis progressed from 1 month $(4.4 \% \pm 0.4 \%)$ to 3 months $(9.2 \% \pm 1 \%)$, along with TGF- $\beta$ signaling and tissue expression of profibrotic miR-21. Although plasma miR-21 was upregulated with diastolic dysfunction, it was downregulated with the onset of systolic dysfunction), correlating with RV fibrosis. Plasma miR-21 in children with PI+PS followed a similar pattern. A model of combined $\mathrm{RV}$ volume and pressure overload recapitulates the evolution of $\mathrm{RV}$ failure unique to patients with prior RV outflow tract surgery. This progression was characterized by enhanced TGF- $\beta$ and miR-21 signaling. miR-21 may serve as a plasma biomarker of RV failure, with decreased expression heralding the need for valve replacement.
\end{abstract}

Conflict of interest: The authors have declared that no conflict of interest exists.

Submitted: November 7, 2016 Accepted: March 23, 2017 Published: May 4, 2017

Reference information: JCI Insight. 2017;2(9):e91625. https:// doi.org/10.1172/ji.insight.91625

\section{Introduction}

With advances in surgical and medical management, there has been a dramatic decline in mortality due to congenital heart disease (CHD) and a similarly dramatic increase in adults living with palliated or repaired CHD, now representing 1.4 million individuals in the US alone, $12 \%$ of whom have severe heart defects (1). Of those with CHD, such as tetralogy of Fallot, pulmonary atresia, or other lesions requiring a right ventricular-to-pulmonary artery (RV-to-pulmonary artery) conduit, one of the most common hemodynamic residua is the combination of pulmonary insufficiency (PI) with mild-to-moderate pulmonary stenosis (PS), leading to a unique combination of chronic RV volume overload and pressure overload, respectively. The incidence of this physiology has increased as surgeons have transitioned away from the traditional large transannular patch approach to smaller patches and valve-sparing techniques in order to relieve RV outflow tract obstruction (RVOTO), while reducing the risk of chronic free PI and RV dilation (2-7). However, over decades, the resultant combination of moderate-to-severe PI plus mild-to-moderate PS results in progressive RV dilation and early RV diastolic dysfunction. Progression to RV systolic failure may take years to develop, but once RV failure ensues it may persist even after pulmonary valve replacement $(2,3)$. Persistence of RV dysfunction may reflect irreversible adverse remodeling associated with chronic volume and pressure overload and may be a precursor to late arrhythmias and sudden death (8). Current decision-making regarding timing of surgical reintervention relies on the use of RV volume and function indices derived from echocardiogram or MRI, and intervention is often delayed, especially in childhood, to avoid the need for serial re-operations due to patient growth $(9,10)$. However, MRI (delayed enhancement, T2 weighting) sensitivity is not sufficient to detect early RV fibrosis, and both echocardiogram and MRI are imperfect measures to predict whether a dilated and hypertrophied RV will remodel and recover function following valve replacement. These issues highlight the need to better understand the mechanisms of RV failure in this unique setting and to identify potential biomarkers to augment cardiac imaging, allowing clinicians to 
plan intervention at an optimal time to preserve long-term RV function. Although there are substantial data on the molecular mechanisms of LV failure due to pressure overload (11-13), there is considerably less known about LV $(14,15)$ or RV (16) volume overload and, as models have been lacking, no data on the mechanisms of RV failure during combined volume and pressure loading.

To address these questions, we developed a murine model of combined moderate-to-severe RV volume overload with mild-to-moderate pressure overload to simulate the residual lesions experienced by patients following $\mathrm{RV}$ outflow tract reconstruction. We determined the degree to which this model recapitulated the physiological changes experienced by patients and used it to evaluate the molecular mechanisms regulating disease progression from an "asymptomatic" stage of diastolic dysfunction to a stage of overt right heart failure with systolic dysfunction. We then identified a candidate biomarker of disease progression, which, if confirmed in large-scale clinical studies, could aid clinicians in the optimal timing for pulmonary valve replacement.

\section{Results}

Model of PI+PS recapitulates the clinical setting - early diastolic dysfunction progressing to late systolic dysfunction. Mice were assessed weekly over a 3-month period after creation of PI and PS (PI+PS). The pulmonary artery band $(\mathrm{PAB})$ gradient and the RV systolic pressure remained stable over time, while the RV internal diameter and RV end-diastolic area progressively increased from 1 month to 3 months (Supplemental Figure 1, F-H; supplemental material available online with this article; https://doi.org/10.1172/jci. insight.91625DS1). Diastolic dysfunction developed starting at 1 month after generation of PI+PS, as evidenced by an increase in the echo Doppler tricuspid valve E/A ratio, consistent with restrictive RV filling (Figure 1, A and B) and elevated RV end-diastolic pressure on catheterization (Figure 1C). Exercise capacity was decreased (Figure 1D); however, there were no overt signs of heart failure. At 2 months, RV diastolic dysfunction was also present, but there were still no signs of RV failure. However, by 3 months, systolic dysfunction had developed, evidenced by decreased RV outflow tract shortening (Figure 1E) and catheterization-derived $\mathrm{dP} / \mathrm{dt}$ (Figure $1 \mathrm{~F}$ ). By this time, all mice had developed overt "clinical" signs of RV failure: edema, tachypnea, and decreased activity (Figure 1, G and H), with a median onset at 77 days (range 62-90 days) following surgery. Edema was assessed by weight and the presence of ascites, while tachypnea was described when there was rapid breathing. Left ventricular (LV) diastolic dysfunction was manifested early, associated with a septal shift, and evidenced by a decrease in the mitral valve inflow Doppler E/A ratio. However, LV systolic function was preserved at all time points, as assessed by LV free wall velocity via tissue Doppler imaging (S') (Supplemental Figure 1, I and J). (Alternative measures of LV function, such as fractional shortening, were not reliable due to distortion of LV geometry by the shift in the septum.) These clinical, echocardiographic, and catheterization data recapitulate the pattern seen in patients with combined volume and pressure overload at various stages of RV failure $(3,8)$.

Progressive increase in $R V$ hypertrophy and subendocardial fibrosis. RV weight was increased at all time points after surgery, while liver weight only increased with the onset of clinical heart failure. LV weight did not increase at any time point. Consistent with the absence of LV dysfunction, lung weight also did not increase at any point (Supplemental Figure 1, K-N). RV myocyte hypertrophy increased dramatically at 1 month, following which the rate of increase in RV myocyte size was less dramatic. This correlated with the afterload (as assessed by PAB gradient and RV systolic pressure) being stable over time, thereby again recapitulating the clinical scenario occurring after tetralogy repair, in which most patients have stable pressure overload in addition to volume overload (Figure 1, I and J). The presence of diastolic dysfunction at 1 month was associated with the early onset of fibrosis, which was initially limited to the RV subendocardium, but which extended to the perivascular region and increased in severity over time (Figure 1, K and L). ImageJ software was used to assess fibrosis in the entire RV. Minimal apoptosis was noted at all time points $(<0.05 \%)$. Thus, we have created a chronic model of combined RV volume and pressure overload that demonstrates early diastolic dysfunction without overt signs of heart failure (compensated stage) and progresses to systolic dysfunction with overt heart failure, recapitulating the long-term clinical course of RV failure in patients with PI+PS. We next used this model to identify candidate pathways mediating the compensated stage of diastolic dysfunction and the transition to systolic dysfunction with clinical heart failure.

Transcriptional profiling of the failing $R V$. During the early "asymptomatic" stage of diastolic dysfunction (1 month), there were surprisingly minimal gene expression changes, a marked contrast to previous studies of isolated PS, isolated PI, or models of LV afterload, such as transverse aortic constriction (TAC) (17-19). Only 

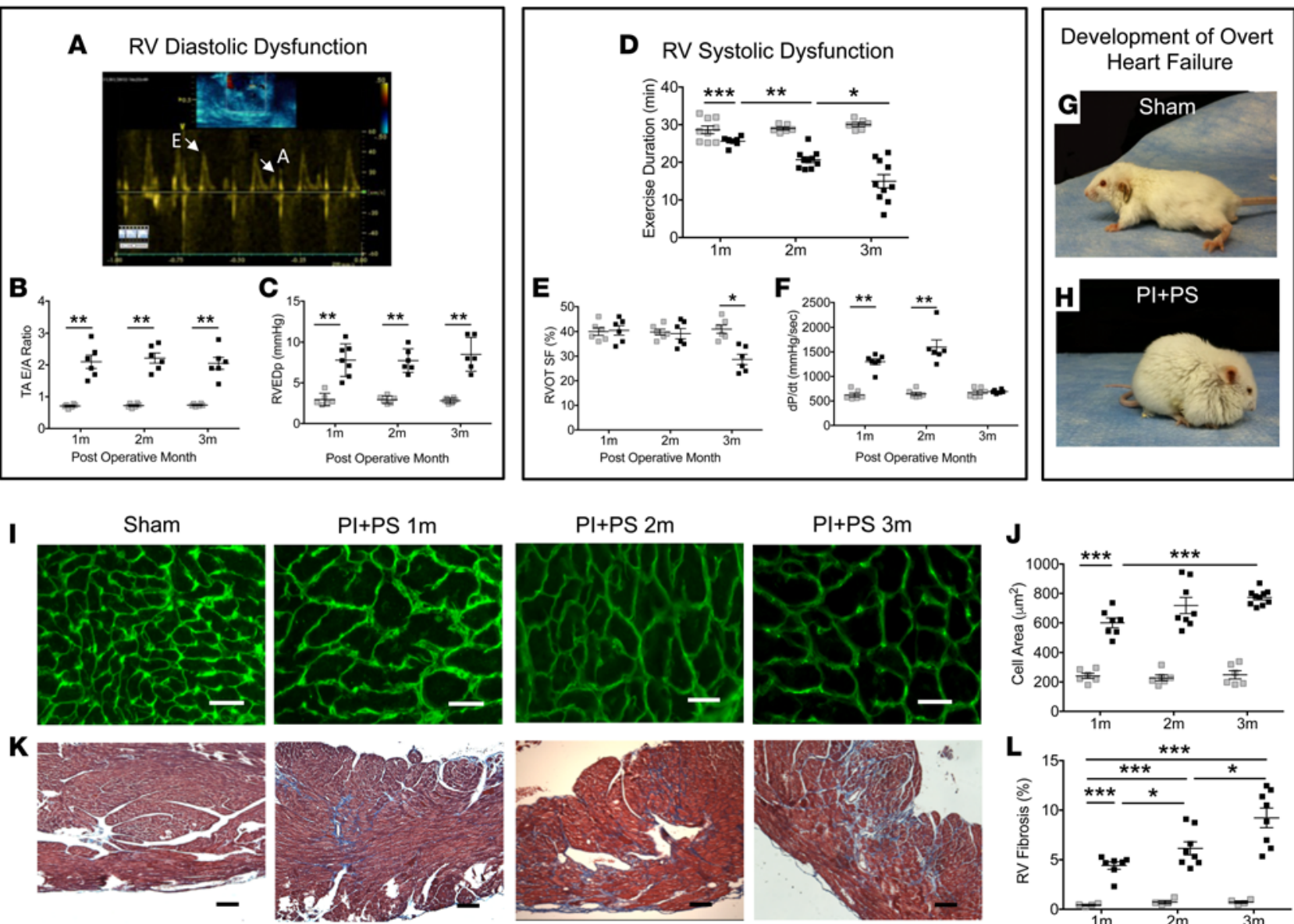

$\square$ Sham

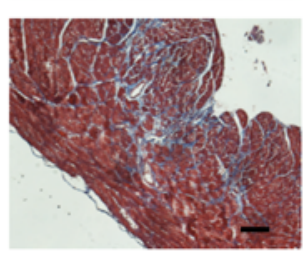

$\mathrm{PI}+\mathrm{PS}$

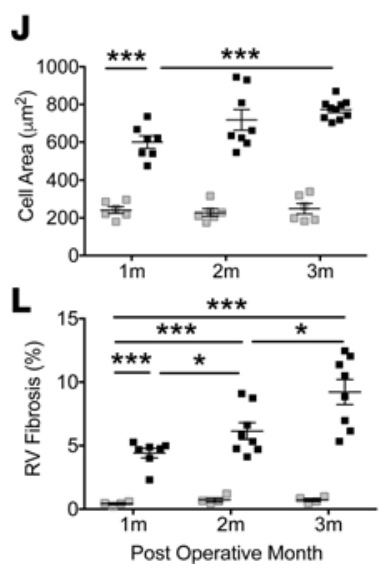

Figure 1. Clinical and histologic characteristics recapitulate the clinical progression of PI+PS. Sham-operated mice and mice with $\mathrm{PI}+\mathrm{PS}$ were assessed using echocardiogram, cardiac catheterization, and exercise testing at 1, 2, and 3 months. Early RV diastolic dysfunction manifested as an increase in (A and B) tricuspid inflow E/A ratio by echocardiogram and (C) RV end diastolic pressures (RVEDp) by cardiac catheterization. Late RV systolic dysfunction characterized by (D) progressive decline in exercise duration by modified treadmill testing, (E) decrease in RV outflow tract (RVOT) shortening fraction (SF) by echocardiogram at 3 months, and (F) decrease in dP/dt by RV catheterization after an early increase. (G and $\mathbf{H}$ ) Development of overt heart failure compared with generalized edema. (I and J) Histologic progression: A progressive increase in myocyte cell area was seen by wheat germ agglutinin staining of the cell wall in green, during the stage of diastolic dysfunction ( 1 month and 2 months) and plateaued with systolic dysfunction ( 3 months). Scale bar: 50 $\mu \mathrm{m}$. (K and $\mathbf{L}$ ) Progressive increase in subendocardial fibrosis was seen with trichrome staining. $n=6-9$ sham for each time point at 1,2 , and 3 months; $n=$ 4-10 PI+PS for each time point at 1, 2, and 3 months. Scale bar: $100 \mu \mathrm{m}$. PI+PS, pulmonary insufficiency and pulmonary stenosis; RV, right ventricle. Data represent mean $\pm \mathrm{SEM}$ and were analyzed using ANOVA with multiple testing correction. ${ }^{*} P<0.05,{ }^{* *} P<0.01,{ }^{* *} P<0.001$.

23 genes were upregulated, including those encoding proteins involved in metabolic (insulin-like receptors, adenylate cyclase), transport (organic anion transporters), and fibrotic (fibroblast growth factors) pathways, while 33 were downregulated, including those encoding proteins involved with pathways related to oxidative stress (NADPH oxidase 4, glutathione transferase) and chemokine response (chemokine ligands, adhesion molecules). In contrast, by 2 months, although systolic function was maintained and there were still no signs of overt heart failure, there was a robust transcriptional response. 583 genes were upregulated, including structural genes, such as myosin- and calcium-binding proteins (myosin-binding protein $\mathrm{H}$, myosin polypeptides, glycoproteins, transmembrane proteins, S100 calcium-binding proteins, calcium calmodulin-dependent protein kinase) and fibrotic (fibroblast growth factors) and stress-responsive genes (integrins, chemokines and cyclins, tumor necrosis factor ligands). 733 genes were downregulated involving metabolic (negative regulation of hydrolase, endopeptidase, peptidase, VLDLP, apolipoproteins, fatty acid-binding protein) and transport genes (Na-K transporting ATPases). With the progression to systolic dysfunction and heart failure (3 months), 863 genes were upregulated, with those encoding proteins involved with inflammatory pathways (chemokines, interleukins, immunoglobulins) increasing for the first time and representing the majority of this late transcriptional response, in addition to ongoing fibrotic pathway upregulation. With the onset of systolic 
failure, metabolic enzymes (NADH dehydrogenase, aldehyde dehydrogenase, Acyl CoA, mitochondrial CoA reductase), transport enzymes (solute carriers), and ion channels (calcium and potassium) were among the principal downregulated pathways (Figure 2A). Thus, transcriptional profiling of the RV during the transition from diastolic to systolic dysfunction revealed a progressive downregulation in metabolic, transport, and ion channels, while structural, inflammatory, and fibrosis-related pathways were upregulated. As expected, the canonical "heart failure" gene ANP was only slightly increased during the stages of diastolic dysfunction and then markedly increased with the onset of systolic dysfunction (6.6 $\pm 4.9-, 8.1 \pm 4.5-$, and $37 \pm 13.3-$ fold versus sham at 1, 2, and 3 months, respectively, with values at 3 months being significantly increased; $P<0.001$ ). Similarly, SERCA was decreased and followed a similar pattern $(1 \pm 0.2-,-1.1 \pm 0.8-$, and $-2.4 \pm 0.6-$ fold vs. sham, with values at 3 months being significantly decreased; $P<0.05$.

$T G F-\beta 1$ pathway is upregulated in the PI+PS RV. Transcriptional pathways mediating fibrosis were dysregulated early in the course of PS+PI, at a time when RV diastolic dysfunction was developing. We focused on the TGF- $\beta 1$ pathway, a previously well-described regulator of fibrosis in the heart (Figure $2 \mathrm{~B}$ ). Thrombospondin-1, an upstream activator of TGF- $\beta 1$ was upregulated during the stages of late diastolic dysfunction ( $2 \pm 0.4$ fold change) and systolic dysfunction $(2.4 \pm 0.5$ fold change) versus sham $(P=0.02)$, while TNF- $\alpha$ was not altered. Although TGF- $\beta 1$ gene expression was not increased, TGF- $\beta 1$ activity was, as evidence by increased phosphorylation of receptor-activated SMADs 2 and $3(0.3 \pm 0.1$ vs. $0.6 \pm 0.1$ vs. $0.8 \pm 0.1$ at 1,2 , and 3 months, respectively, with values at 2 and 3 months being significantly increased; $P<0.02$ ) (Figure 2C), while the expression of inhibitors of fibrosis, such as SMAD7 and Sprouty, was decreased (Figure 2, D-F).

$T G F-\beta$ regulated profibrotic miRs are upregulated in the PI+PS RV. We next evaluated the expression of profibrotic miRs regulated by TGF- $\beta$, miR-21, miR-21*, the miR-221/222 cluster, and miR-143, to further define the mechanism of RV fibrosis. RV free wall miR-21 increased 2.5-fold starting at the stage of early diastolic dysfunction ( 1 month) and further increased by 4 -fold at the stage of late diastolic dysfunction ( 2 months). With the onset of systolic dysfunction ( 3 months), miR-21 remained upregulated, but less so than at 2 months (Figure 3A). miR-21 expression positively correlated with the degree of RV fibrosis during the phase in which diastolic dysfunction progressed (miR-21 $\mathrm{r}=0.76$, $P=0.0002$ ) (Figure 3B); however, this correlation lessened once systolic dysfunction developed, since fibrosis continued to increase but the tissue expression of miR-21 did not (miR-21 $\mathrm{r}=0.51, P=0.003)$. At the time of RV failure, miR-21 was only being produced by the heart and not by the liver, spleen, or lungs (0.9-1.4-fold vs. sham, $P=\mathrm{NS}$; Figure 3C). We next determined the source of cardiac miR-21, demonstrating that the majority of miR-21 was derived from the noncardiomyocyte fraction (fibroblasts and endothelial cells) instead of from cardiomyocytes ( $85 \pm 14-$ vs. $4 \pm 1$-fold, $P<0.001$ ) (Figure 3D and Supplemental Figure 3). Upregulation of miR-21 was associated with a decrease in expression of its target proteins Smad 7 and Sprouty 1, which mediate ECM synthesis, although PTEN, which is also a miR-21 target protein mediating ECM degradation and apoptosis, was unchanged. The other profibrotic miRs $21^{*}, 221$ and 222, were also upregulated at all time points (Supplemental Figure $2, \mathrm{~A}-\mathrm{C}$ ). The overall expression of miR-21* passenger strand (miR-21-3p) was lower than the other miRs, which may reflect intracellular degradation of the passenger strand, as described by Bang et al. (20). miR-143 was not significantly different from sham controls at any time point.

Plasma miR-21 is upregulated with diastolic dysfunction but switches to being downregulated with the development of systolic dysfunction. Circulating miRs in body fluids have been described as biomarkers of cardiovascular disease and cancer progression (21-23). We therefore evaluated the expression of the upregulated RV profibrotic miRs in plasma to evaluate their usefulness as potential biomarkers of fibrosis in the RV. Plasma miR expression was profiled from PI+PS and sham controls along with matched tissue samples at all time points. Whereas the RV expression of miR-21 increased at all time points, plasma expression increased with early and late diastolic dysfunction but then transitioned to being downregulated in the plasma with the onset of systolic dysfunction, at a time at which it was still increased in the heart (Figure 3E). During the stage of diastolic dysfunction, the increase in plasma miR-21 correlated positively with RV size $(\mathrm{r}=0.64, P<0.001)$ and the degree of RV fibrosis $(\mathrm{r}=0.71$, $P<0.0001$ ) (Figure 3, I and K). However, with the onset of systolic dysfunction, plasma miR-21 levels began to decrease, while RV fibrosis continued to increase. There was no correlation with RV size (Figure 3, J-L). Plasma expression of miR-221 and miR-222 was decreased ( $>2$-fold vs. sham) at all time points, while their RV expression was upregulated (Supplemental Figure 2, D and E). miR-21*, however, was not detectable in the plasma, despite tissue level upregulation, which may be related to rapid 


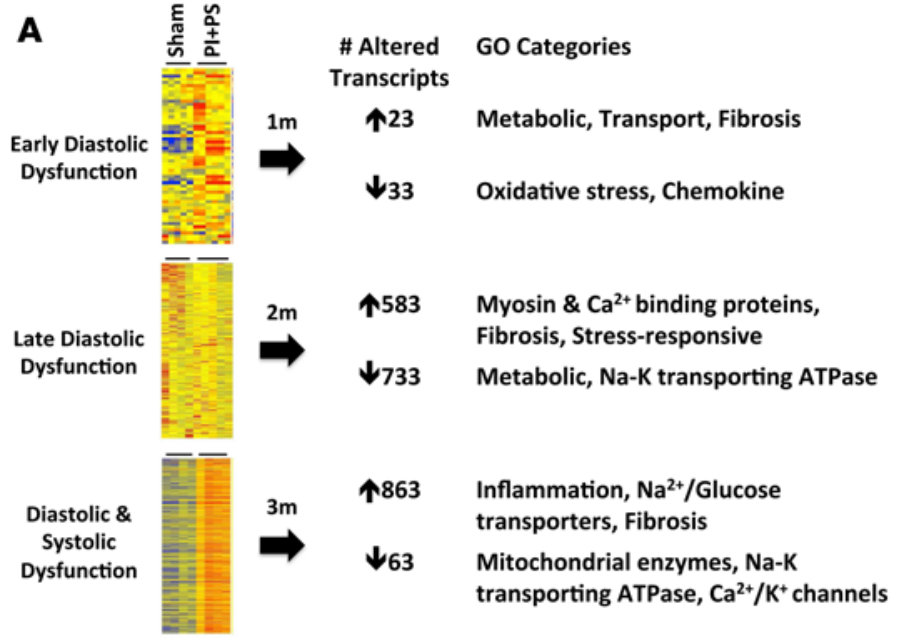

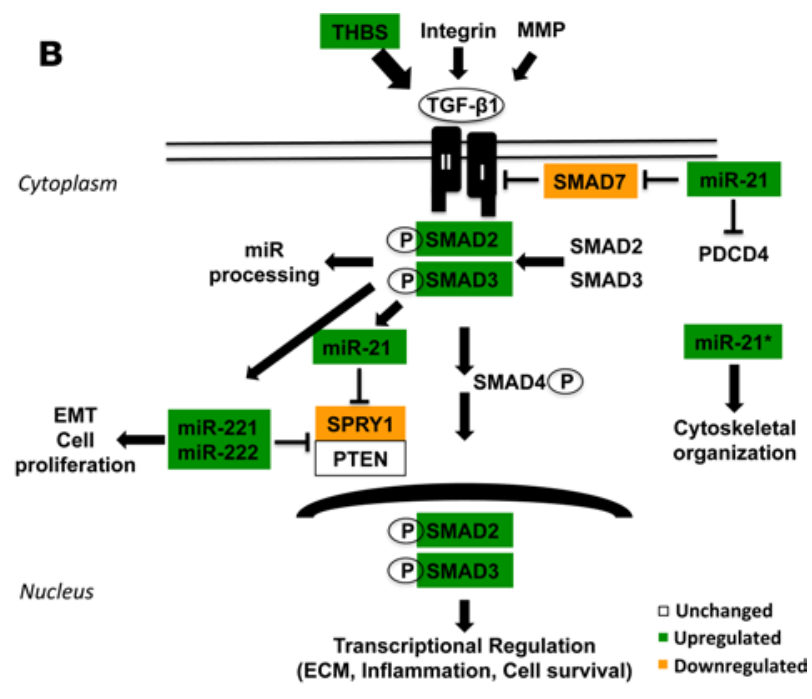

E
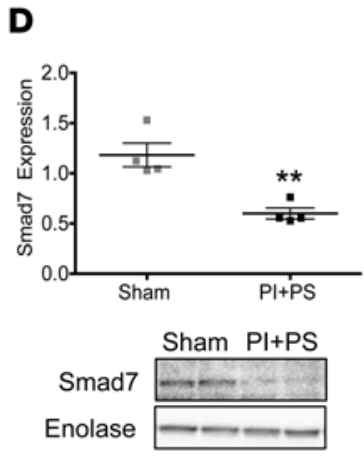
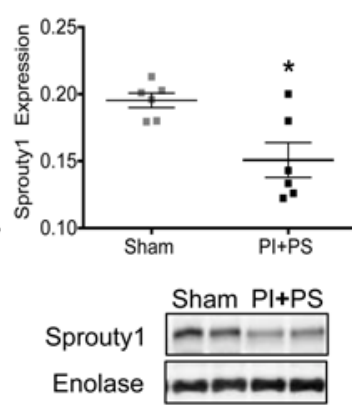

$\mathbf{F}$
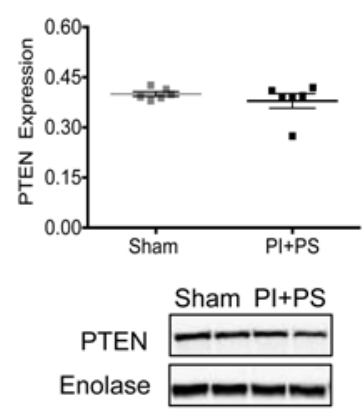

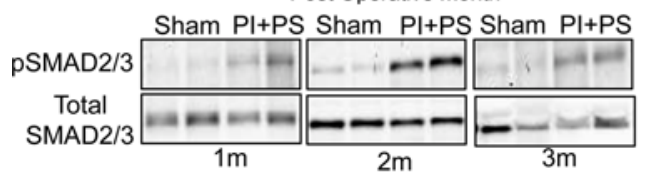

$\square$ Sham $\square \mathrm{PI}+\mathrm{PS}$

Figure 2. TGF- $\beta$ pathway is upregulated with PI+PS. (A) Transcriptional profiling of the failing right ventricle. Agilent whole-genome oligonucleotide microarrays were used to evaluate gene expression at 1 and 2 months (diastolic dysfunction) as well as 3 months (systolic dysfunction) following surgery and compared with sham-operated controls. Metabolic, transport, and ion channel pathways were downregulated over time, while structural, inflammation, and fibrosis-related pathways were upregulated (fold change $\geq 2$, Benjamini-Hochberg multiple testing correction with corrected $P<0.05$ ). $n=4-6$ / sham and $\mathrm{PI}+\mathrm{PS} /$ time point. (B) Schematic of TGF- $\beta$ signaling and its regulation of profibrotic miRs. White box, unchanged protein expression; green box, upregulated protein and miR expression; orange box, downregulated protein expression. (C) Phosphorylated/total SMAD2/3 protein expression is upregulated in $\mathrm{PI}+\mathrm{PS}$ during the stages of diastolic (1 month and 2 months) and systolic dysfunction (3 months). ( $\mathbf{D}$ and $\mathbf{E}$ ) miR-21 downstream target proteins involved in ECM regulation, Smad 7 and Sprouty 1, were downregulated as miR-21 expression increased, while (F) PTEN expression was not changed. $n=$ 4-6/sham and PI+PS. PI+PS, pulmonary insufficiency and pulmonary stenosis; ECM, extracellular matrix. Data are presented as mean \pm SEM. Microarray data was analyzed using GeneSpring GX 11.5 software. An unpaired, 2-tailed Student's $t$ test was utilized for 2-group comparisons and ANOVA with multiple testing correction for 3 or more group comparisons. ${ }^{*} P<0.05,{ }^{* *} P<0.01$.

degradation in the heart, as reported by Bang et al. (20). To eliminate the possibility that the decrease in plasma miRs was due to a change in distribution between plasma and serum (24), we confirmed that the serum expression of miR-21 and miR-221/222 was similar to that in plasma.

miR-21 is transported via exosomes to the plasma. We next investigated the mechanism for this intriguing divergence between plasma and tissue miR expression, a phenomenon that has been previously described in cancer $(23,24)$. We examined whether miR transport from the heart to the plasma was altered with the onset of systolic RV failure, focusing on miR-21, since it showed high concentration in the plasma, was easily detectable, and its expression changed dramatically with disease progression, thereby giving it the greatest potential to serve as a biomarker. We began with an evaluation of exosome transport of miR-21 from cardiac fibroblasts to the bloodstream, similar to that described in hepatocellular carcinoma and other cancers (25). Plasma exosomes were identified via transmission electron microscopy imaging (Figure 3F) and expressed CD63, a fibroblast-derived exosomal marker confirming the fibroblast origin of these exosomes (Figure 3G). Exosomal marker expression and exosomal abundance were similar in sham controls and during both the 
A
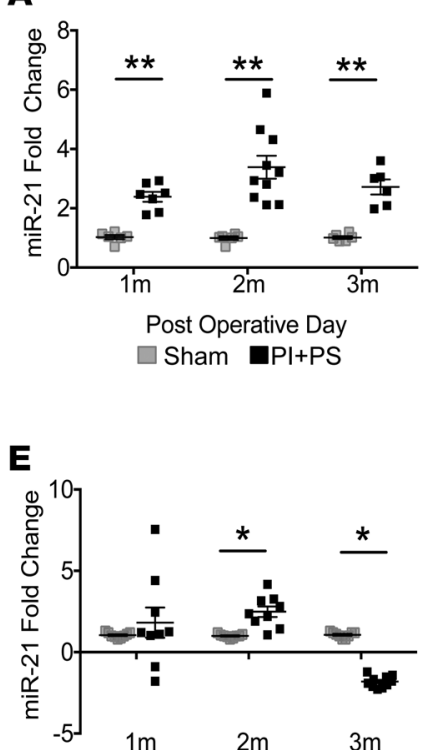

Post Operative Month

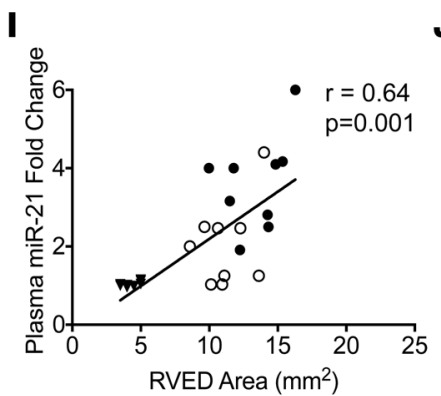

B

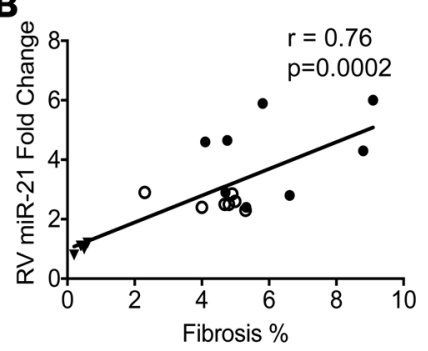

\section{$\mathbf{F}$}

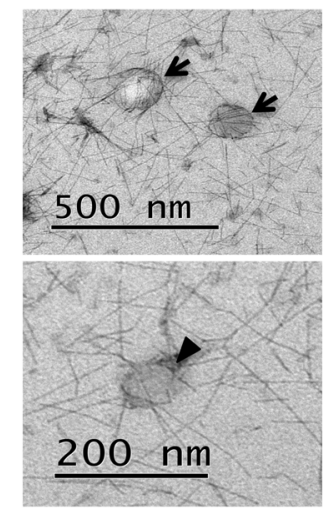

J

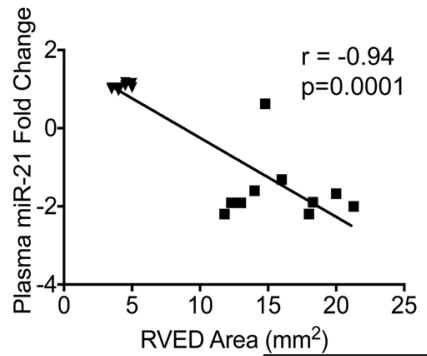

C

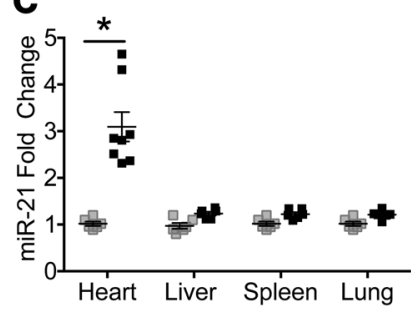

G
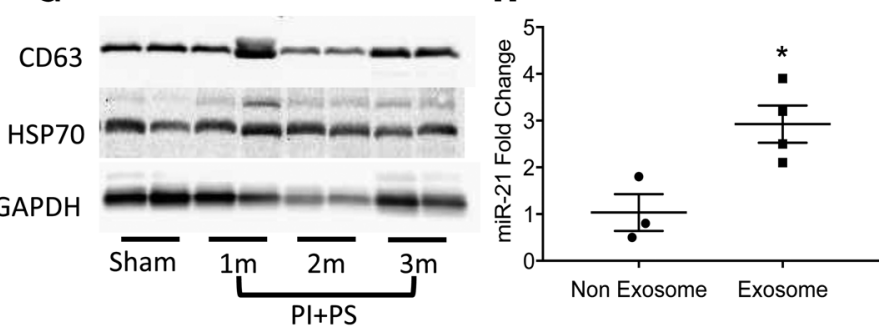

K

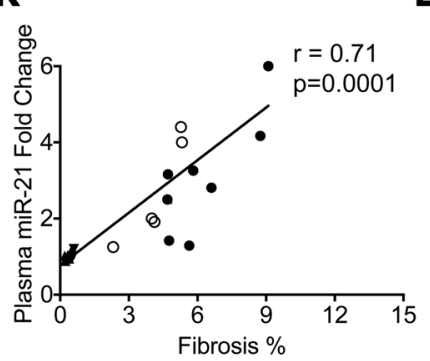

D

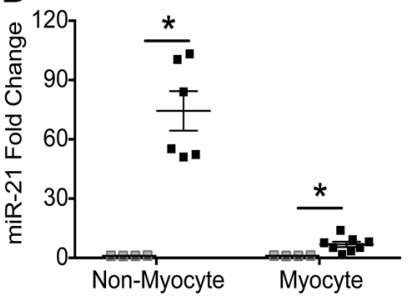

$\nabla$ Control; o 1m; $2 \mathrm{~m} ; \mathbf{\square} 3 \mathrm{~m}$

Figure 3. $\mathbf{m i R}-\mathbf{2 1}$ is a biomarker of $\mathbf{R V}$ dysfunction in mice. (A) RV expression of profibrotic miR-21 was upregulated during the stages of diastolic (1 month and 2 months) and systolic (3 months) dysfunction compared with sham-operated controls. $n=6-8 /$ sham and PI+PS/time point. (B) The expression of miR-21 positively correlated with the degree of RV fibrosis as diastolic dysfunction progressed, but that correlation was lost once systolic dysfunction developed. (C) miR-21 expression is upregulated in the hearts in PI+PS mice and not in the livers, spleens, or lungs. $n=5 / \mathrm{sham} ; n=8 / \mathrm{PI}+\mathrm{PS}$. (D) miR-21 is expressed predominantly in nonmyocytes. $n=4 /$ sham; $n=6-8 / \mathrm{PI}+\mathrm{PS}$. (E) Plasma miR-21 was upregulated with diastolic dysfunction but switched to being downregulated with the development of systolic dysfunction, despite persistent tissue-level upregulation ( $n=9-10 /$ sham and PI+PS/time point); miR-21 is expressed in plasma exosomes. (F) TEM demonstrating exosomes (arrows) 50-100 nm in size, with characteristic cup shape (arrowhead) and surrounding halo. Scale bar: $500 \mathrm{~nm}$ (top); $200 \mathrm{~nm}$ (bottom). (C) Exosome marker expression was similar at all time points. $n=2 / \mathrm{group}$. (H) miR-21 is enriched in exosomes in the plasma compared with the nonexosome fraction. $n=3 /$ sham; $n=4 / \mathrm{PI}+\mathrm{PS}$. (I) Plasma miR-21 correlated with RV end-diastolic area (RVED) as diastolic dysfunction progressed and (J) switched to a negative correlation with the onset of systolic dysfunction. (K and $\mathbf{L}$ ) A similar correlation was seen with fibrosis. RV, right ventricle; $\mathrm{PI}+\mathrm{PS}$, pulmonary insufficiency and pulmonary stenosis; TEM, transmission electron microscopy. Data are presented as mean \pm SEM. An unpaired, 2-tailed Student's $t$ test was utilized for 2-group comparisons and ANOVA with multiple testing correction for 3 or more group comparisons. Correlation was assessed using Pearson's correlation. ${ }^{*} P<0.02,{ }^{* *} P<0.001$.

phases of diastolic and systolic dysfunction, indicating that exosomal formation, cellular release, and transport are preserved during RV failure and are not the etiology of the decrease in plasma miR levels. Exosome miR-21 expression was 3-fold greater than in the nonexosomal plasma fraction (Figure $3 \mathrm{H}$ ), demonstrating that miR-21 is enriched in plasma exosomes. In contrast, miR-221 and miR-222 were undetectable in exosomes, indicating that they are not transported via this mechanism. In summary, miR-21 is transported to the plasma in fibroblast-derived exosomes. miR-21-containing exosome formation, release, and transport are intact in RV failure. Thus, the decrease in plasma miR-21 may be due to a defect in the intracellular incorporation of miR-21 into exosomes and/or to increased intracellular degradation.

$R V$ miR-21 is upregulated in children with PI+PS and is associated with increasing fibrosis. To determine whether our finding of increased profibrotic miRs was seen in children with CHD, we assessed myocardial miR-21 
expression and RV fibrosis where RV infundibular muscle bundles were resected as part of the surgery in (a) acyanotic infants undergoing initial surgery for tetralogy of Fallot $(n=7)$, (b) children with tetralogy undergoing repeat surgery for residual volume and pressure overload lesions with preserved RV function by echo ( $n$ $=4$ ), and (c) explanted hearts from children with dilated cardiomyopathy at the time of cardiac transplantation with RV failure. Children undergoing surgery for mild RVOTO due to double-chambered RV with tiny, restrictive ventricular septal defect $(n=3)$ were controls. Baseline characteristics and echocardiographic data are shown in Table 1. Of note, controls and children with residual volume and pressure overload were age matched. While there was minimal upregulation of miR-21 in children with unrepaired tetralogy compared with those with mild RVOTO, there was progressive upregulation with PI+PS and with end-stage RV failure $(1.6 \pm 0.1-, 1.29 \pm 0.1-, 3.1 \pm 0.4-, 5.2 \pm 1.2-$ fold, respectively, $P<0.0001)$ (Figure 4A) and a progressive increase in fibrosis $(1.3 \% \pm 0.2 \%, 2.54 \% \pm 0.3 \%, 5.63 \% \pm 0.6 \%, 18.54 \% \pm 0.5 \%$, respectively, $P<0.0001)$. Increasing RV miR-21 expression correlated with increasing fibrosis $\left(\mathrm{r}^{2}=0.66, P<0.01\right)$ (Figure $4, \mathrm{~B}$ and $\mathrm{C}$ ).

Plasma miR-21 is decreased in children with severe PI/mild-to-moderate PS. To determine whether our findings in the murine PI+PS model were translatable to humans with PI+PS, we next performed a pilot study to evaluate plasma miR-21 expression in children with residual lesions long term following surgery for tetralogy of Fallot. Two groups of tetralogy patients were studied: asymptomatic patients with mildly enlarged RVs (RV end-diastolic volume [RVEDV] by MRI $113.5 \pm 33.2 \mathrm{ml} / \mathrm{m}^{2}$ ) and normal RV ejection fraction $(n=7)$ and symptomatic patients with exercise intolerance, moderate-to-severe RV enlargement (RVEDV by MRI $150.2 \pm 21.8 \mathrm{ml} / \mathrm{m}^{2}$ ), and decreased RV ejection fraction; the later group was analyzed at the time of admission for pulmonary valve replacement $(n=9)$. These two groups were compared with healthy control children $(n=10)$. (Baseline characteristics and echocardiographic and cardiac MRI data are shown in Table 2.) This is a separate group of patients compared with those shown in Table 1, since it is not always possible to obtain matched samples. In agreement with our animal model, plasma miR-21 was upregulated during the asymptomatic phase $(2.3 \pm 0.4$-fold, $P<0.01)$ and progressed to being downregulated during the symptomatic phase versus controls $(-3.1 \pm 0.4$-fold, $P<0.001)$ (Figure 5A). Plasma miR-21 levels decreased as RV size increased $(\mathrm{r}=-0.67, P<0.01)$ and RV function decreased $(\mathrm{r}=0.74, P$ $=0.0005$ ) (Figure 5, B and C). We isolated exosomes from human plasma and confirmed their fibroblast origin (Figure 5, D-F), similar to that in our murine model. Plasma miR-21 expression was enriched 8-fold in exosomes versus the nonexosome fraction (Figure 5G). Plasma miR-221 and miR-222 were also downregulated and miR-21* was minimally expressed at $\mathrm{Ct}>35$ (data not shown).

\section{Discussion}

Combined chronic RV volume and pressure overload is a unique hemodynamic sequela seen in children and adults with complex CHD following RV outflow tract reconstruction. Evolution in surgical methods over the last decade, aimed at preserving pulmonary valve integrity, have increased the occurrence of this combined lesion, whose hemodynamic consequences and long-term effects on RV function have yet to be determined. This is a critical issue, as more children with complex CHD are surviving into adulthood, many of whom are at risk for chronic complications, such as arrhythmias and heart failure (9, 26-28). Although pulmonary valve replacement can ameliorate the combined volume and pressure loads, reduce RV dimension, and improve New York Heart Association functional class, surgical intervention has not necessarily improved exercise capacity, arrhythmia burden, ejection fraction, or survival, demonstrating the large gaps in our knowledge about how best to manage this rapidly growing group of patients $(2,10,26$, 28). RV fibrosis in this patient population is associated with worsening symptoms, exercise intolerance, and ventricular dysfunction (29). Thus, increasing our understanding of the molecular changes mediating the transition from compensated RV diastolic dysfunction to symptomatic RV systolic dysfunction in patients with this unique physiology has the potential to aid clinicians in the more optimal timing of intervention to allow for best preservation of RV function and reversal of adverse remodeling.

Here, we present a murine model of chronic RV volume and pressure overload that recapitulates many aspects of the clinical condition, with early diastolic dysfunction progressing to late systolic dysfunction. In contrast, a murine model of pure volume overload results in diastolic dysfunction without evidence of systolic dysfunction over a similar time period, highlighting the RV's ability to adapt more favorably to volume overload, as compared with combined volume and pressure overload. The advantage of our PI+PS model over prior models of isolated PS or PI $(16,17)$ is that it more accurately recapitulates the actual hemodynamic loads and the more chronic clinical progression of this disease in humans, in partic- 
Table 1. Patient characteristics of children with mild RV outflow tract obstruction, tetralogy of Fallot, $\mathrm{PI}+\mathrm{PS}$, and end-stage heart failure

\begin{tabular}{lccccc}
\hline Characteristics & $\begin{array}{c}\text { Mild RVOTO } \\
(\boldsymbol{n}=\mathbf{3})\end{array}$ & $\begin{array}{c}\text { Unrepaired tetralogy } \\
\text { of Fallot }(\boldsymbol{n}=\mathbf{7})\end{array}$ & $\begin{array}{c}\mathbf{P I + P S} \\
(\boldsymbol{n}=\mathbf{4})\end{array}$ & $\begin{array}{c}\text { End-stage heart failure } \\
(\boldsymbol{n}=\mathbf{2})\end{array}$ & $\boldsymbol{P}$ value \\
Age (yr) & $2.9 \pm 1.2$ & $0.3 \pm 0.05$ & $3.4 \pm 1.3$ & $7.7 \pm 4.1$ & $<0.05$ \\
Male (\%) & 67 & 57 & 25 & 100 & $<0.05$ \\
Degree of PI & None & None & Severe & None & - \\
PS gradient (mmHg) & $30.7 \pm 3$ & $93.9 \pm 7.9$ & $83.8 \pm 4.8$ & $8 \pm 4$ & $<0.01$ \\
RV FAC (\%) & $48 \pm 5.2$ & $52 \pm 4.1$ & $54 \pm 7$ & $18 \pm 7.5$ & $<0.01$
\end{tabular}

$\mathrm{RV}$, right ventricle; $\mathrm{PI}+\mathrm{PS}$, pulmonary insufficiency and pulmonary stenosis; RVOTO, RV outflow tract obstruction; FAC, fractional area change.

ular the progressive exercise intolerance and RV dilation, thereby enhancing the translation of molecular remodeling mechanisms to patients. More commonly used animal models of load-induced heart failure, e.g., acute severe LV afterload stress (TAC) or acute severe RV afterload stress (PS) are uncommon in children or adults with repaired $\mathrm{CHD}$, and therefore data from these models may not be clinically relevant to this population. During the stage of early diastolic dysfunction, transcriptional profiling shows activation of pathways regulating fatty acid synthesis and transport enzymes for the generation of increased ATP to meet the increased metabolic demands of the preloaded and postloaded heart. This is in stark contrast to our murine model of RV volume overload, in which none of these pathways were upregulated. Fibroblast growth factor pathways were also upregulated during this stage, a time during which early subendocardial fibrosis was detected. In contrast to this earlier stage, the stage of late diastolic dysfunction was marked by changes in gene pathways regulating structural remodeling. This was associated with myocyte hypertrophy and increasing subendocardial fibrosis that at this time began to expand transmurally. Again, this is in contrast to isolated RV volume overload, in which the TGF- $\beta$ pathway is not significantly upregulated and there is dramatically less fibrosis, which is totally limited to the subendocardium. In PI+PS, important mediators of the fatty acid cycle, such as fatty acid-binding proteins, were downregulated, suggesting a switch in energy production away from fatty acids, as has been described in other models of early heart failure (30). Finally, the stage of overt heart failure was notable for upregulation of inflammatory pathways and further upregulation of mediators of fibrosis, a downregulation in mitochondrial enzymes suggesting impaired ATP production, and impaired transport enzymes and ion channels, in particular those related to $\mathrm{Ca}^{2+}$ transient regulation. Chronic volume overload displays many of these changes, with upregulation in inflammation and fibrosis and impaired $\mathrm{Ca}^{2+}$ signaling, but to a much lesser extent. However, chronic volume overload was not characterized by decreased expression of mitochondrial enzymes and transport proteins, which may explain the less severe phenotype and the lack of systolic heart failure in pure PI. These changes highlight the extensive adverse remodeling that occurs even during the asymptomatic stages of PI+PS, prior to the development of systolic dysfunction and clinical heart failure. While the profibrotic remodeling alone is unlikely to fully explain the progression to systolic heart failure, both animal and human studies $(29,31-33)$ show that it is a significant contributing factor. RV volume and pressure overload can also occur in the setting of flow-induced pulmonary hypertension (PAH) due to systemic to pulmonary artery shunts in CHD. Animal models recapitulating this unique pathophysiology demonstrate early alterations in endothelin receptors, endothelial nitric oxide, and pulmonary and systemic vascular inflammatory changes, which may adversely affect RV function (34). However, these models require an additional endothelial insult to develop $\mathrm{PAH}$, such as monocrotaline, and therefore do not really recapitulate the volume- and pressure overload-induced RV dysfunction seen in after tetralogy repair.

RV fibrosis demonstrated by cardiac MRI using late Gadolinium enhancement is a unique feature following RVOT reconstruction; it is seen in $>98 \%$ of children and adults with repaired tetralogy of Fallot and is a precursor to late arrhythmias, exercise intolerance and ventricular dysfunction $(29,31,32)$. Although the mechanisms mediating fibrosis have been described in the postloaded LV (33), whether these mechanisms are similar in the RV subjected to combined volume and pressure load has not previously been evaluated. We identified profibrotic pathways involving TGF- $\beta$ signaling and profibrotic miRs interacting with the TGF- $\beta$ pathway as primary mediators of RV fibrosis in PI+PS. TGF- $\beta$ stimulation also aids in miR processing by 
A

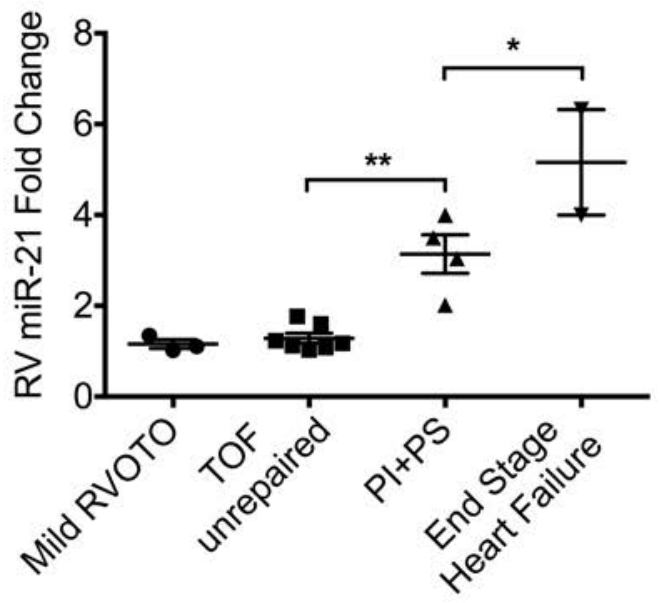

B

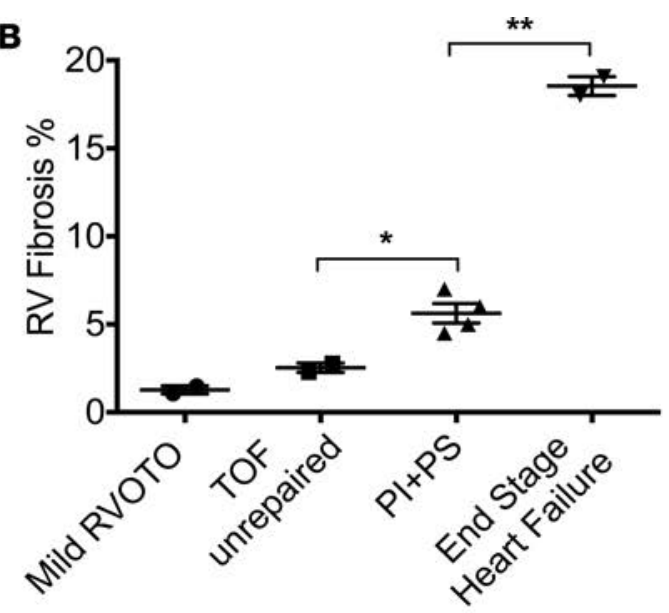

C

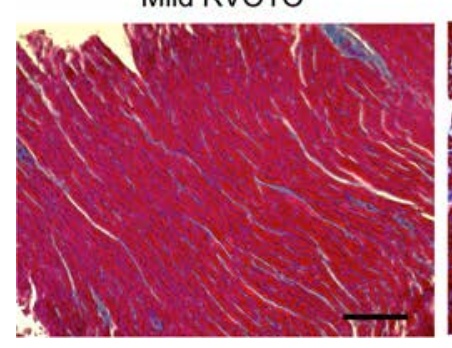

TOF unrepaired

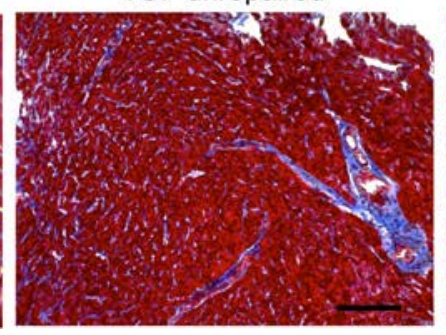

PI+PS

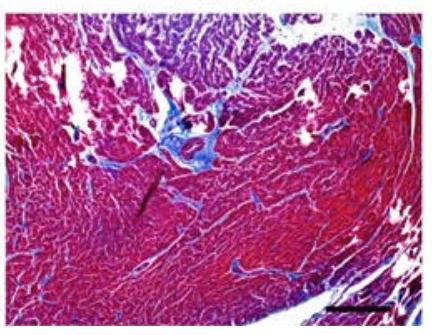

End Stage Heart Failure

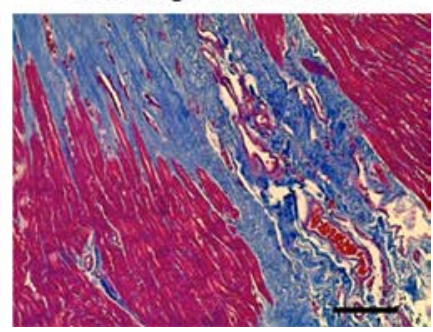

Figure 4. RV miR-21 is upregulated in children with PI+PS and is associated with increasing fibrosis. (A) RV miR-21 expression is upregulated in children with PI+PS $(n=4)$ and end-stage RV failure $(n=2)$ compared with those with mild RVOTO $(n=3)$ and infants with unrepaired TOF $(n=7)$. (B and C) Increasing fibrosis is seen in children with PI+PS $(n=4)$ and end-stage RV failure $(n=2)$. Scale bar: $100 \mu \mathrm{m}$. RV, right ventricle; RVOTO, RV outflow tract obstruction; TOF, tetralogy of Fallot; PI+PS, pulmonary insufficiency and pulmonary stenosis. Data are presented as mean \pm SEM. ANOVA with multiple testing correction was used. Correlation was assessed using Pearson's correlation. ${ }^{*} P<0.01,{ }^{* *} P<0.001$.

activating DROSHA and DICER enzymes and increasing the formation of mature miRs (35). Interestingly, whereas TGF- $\beta$ expression and fibrosis steadily increased with the progression to heart failure, the tissue expression of many of the profibrotic miRs increased with diastolic dysfunction and then leveled off with the onset of systolic dysfunction. One in particular was miR-21. In our model of combined volume and pressure overload, miR-21 upregulation was associated with the induction of matrix protein synthesis via TGF- $\beta$ / SMAD7 and growth factor secretion via SPRY1 but did not inhibit PTEN and PCDC4, suggesting that fibroblast and cardiomyocyte survival and ECM turnover were unaffected (20,36-39). The role of miR-21 in the heart has been controversial. Early reports in TAC have shown that miR-21 originates from fibroblasts alone, thereby playing a role in fibrosis and the progression to LV failure (40). Others report miR-21 release from cardiomyocytes and endothelial cells, albeit to a lesser extent than from fibroblasts, which thereby induces cell hypertrophy, apoptosis, endothelial-to-mesenchymal transition, and arterial remodeling (36). In contrast, knocking out miR-21 does not prevent fibrosis and leads to early heart failure and death, suggesting that miR-21 may have both deleterious and protective effects (37). Whether this is due to a direct effect or whether miR-21 is merely a secondary maker of these events remains to be determined; however, our data suggest that miR-21 could be a useful biomarker for fibrosis and the progression from RV stability to RV failure (41). Drake et al. describe a decrease in RV miR-21 in rats following Sugen/hypoxia and in PAB with hypoxia. These differences may be related to a difference in species and models subject to hypoxia (42).

A major challenge for those caring for both children and adults with CHD is to identify the optimal timing of intervention, such as pulmonary valve replacement, before the development of irreversible damage to the volume- and pressure-loaded RV. miRs are excellent biomarker targets, since they circulate in the bloodstream in a stable, extracellular form, are easily detectable, and have been shown to change with disease course $(43,44)$. Of the upregulated profibrotic miRs in the RV, miR-21 emerges as a potential biomark- 
Table 2. Patient characteristics of controls and those with severe PI and mild-to-moderate PS

\begin{tabular}{|c|c|c|c|c|}
\hline Characteristics & $\begin{array}{l}\text { Controls } \\
(n=10)\end{array}$ & $\begin{array}{l}\mathrm{PI}+\mathrm{PS} \\
(n=7)\end{array}$ & $\begin{array}{l}\text { PI+PS heart failure } \\
\qquad(n=9)\end{array}$ & $P$ value \\
\hline Age (yr) & $14.5 \pm 3.6$ & $10.5 \pm 4.5$ & $8.7 \pm 5.6$ & $<0.01$ \\
\hline Male (\%) & 40 & 50 & 67 & NS \\
\hline PS gradient $(\mathrm{mmHg})$ & 0 & $24.5 \pm 10.6$ & $27 \pm 8$ & $<0.0001$ \\
\hline Pulmonary regurgitant fraction (\%) & 0 & $23.5 \pm 2.1$ & $41.8 \pm 1.9$ & 0.0008 \\
\hline RV end-diastolic volume $\left(\mathrm{ml} / \mathrm{m}^{2}\right)$ & $79.8 \pm 1.3$ & $113.5 \pm 33.2$ & $150.2 \pm 21.8$ & $<0.0001$ \\
\hline RV ejection fraction (\%) & $59.1 \pm 5.8$ & $57 \pm 1.4$ & $44.8 \pm 4.3$ & 0.01 \\
\hline
\end{tabular}

er candidate. miR-21 has been previously described as a biomarker for atherosclerosis and heart transplant rejection and in multiple cancers $(22,24,25,45,46)$. We found that plasma miR-21 increased early during the stage of diastolic dysfunction and positively correlated with the degree of tissue fibrosis. However, with the onset of systolic dysfunction, plasma miR-21 expression switched to being decreased, at a time when tissue miR-21 was still elevated. Thus, the expression of miR-21, -21*, -221, and 222 in the RV of PI+PS is similar to that seen in the LV during pressure overload, except at the time of systolic dysfunction when miR-21 overexpression begins to decrease in the RV but continues to increase in the LV after TAC $(35,47)$. Similar discrepant expression of miRs in tissue versus plasma has been described in other diseases (44). The mechanism of miR transport into the plasma is only now being elucidated and has not been evaluated in heart failure. miRs are transported either encapsulated in vesicles (exosomes, apoptotic bodies, or other microvesicles) or nonencapsulated and bound to RNA-binding proteins (argonaute2 proteins [Ago2]) or lipoprotein complexes (HDL) (43). The relative contribution of each fraction is controversial. Mitchell et al. have shown that the majority of circulating human miRs are bound to Ago 2 complexes, while a minority $(15 \%)$ are associated with exosomes $(24,48,49)$. In contrast, Gallo et al. show that the majority of circulating human miRs are concentrated in exosomes, suggesting that assessing miRs in total serum/plasma may yield false-negative results (50). These conflicting results may be related to differences in isolation techniques resulting in breakdown of exosomes or due to differences in disease states. Circulating human miR-21 is transported in a free circulating form bound to Ago2 $(24,51)$. In contrast, in hepatocellular carcinoma, circulating miR-21 is enriched in the exosome fraction, as in other cancers (25). In our model of RV failure, miR-21 is expressed primarily in cardiac fibroblasts and is exported to the circulation via exosomes. The decrease in miR-21 expression with the onset of systolic dysfunction, at a time when tissue expression is still upregulated, suggests a potential defect in intracellular degradation, exosome packaging of miR-21, or exosome transport. Of these, the only mechanism that could be assessed in vivo is exosome transport. We show that miR-21 continues to be produced by the heart and that its transport mechanism is intact, suggesting increased degradation or a defect in intracellular exosomal packaging of miR-21 as potential mechanisms for the decrease in plasma levels during heart failure.

Finally, we have shown for the first time to our knowledge that increasing RV miR-21 in children with $\mathrm{PI}+\mathrm{PS}$ is associated with increasing fibrosis. In addition, a pilot validation study in children with PI+PS undergoing pulmonary valve replacement demonstrated miR-21's potential utility in monitoring RV dilation and the onset of systolic dysfunction in patients with PI+PS. The true value of this biomarker, and how much it adds to the power of known MRI measures of RV function (RVED volume, RV mass/volume index, delayed enhancement), remains to be determined from future large multicenter studies.

Conclusions. We describe a model of chronic RV volume and pressure overload with early diastolic and late systolic dysfunction, providing investigators with a valuable tool for further evaluation of a form of RV failure that is unique to children and adults with repaired CHD. We identify the TGF- $\beta$ signaling pathway and associated profibrotic miRs, such as miR-21, as mediators of fibrosis in PI+PS, the fibroblast origin of plasma miR-21, and its mechanism of transport to the circulation. Finally, we demonstrate that miR-21 is a potential biomarker of RV failure, with an increase during the phase of diastolic dysfunction indicating early RV fibrosis and a decline in plasma expression heralding the onset of systolic dysfunction. This biomarker has the potential to be used in conjunction with cardiac MRI when assessing the need for valve replacement. 
A

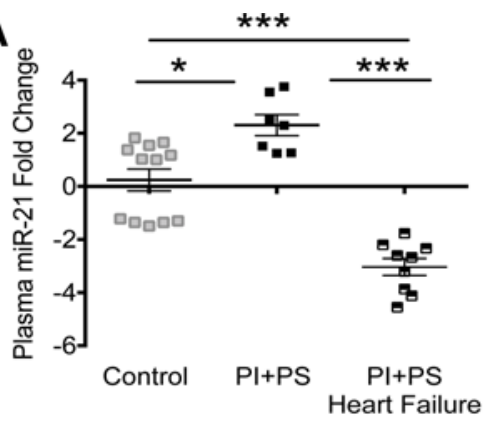

B

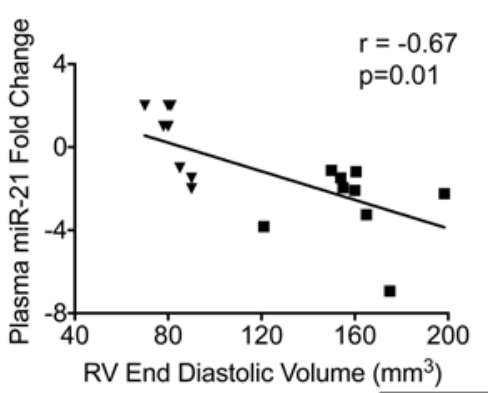

C

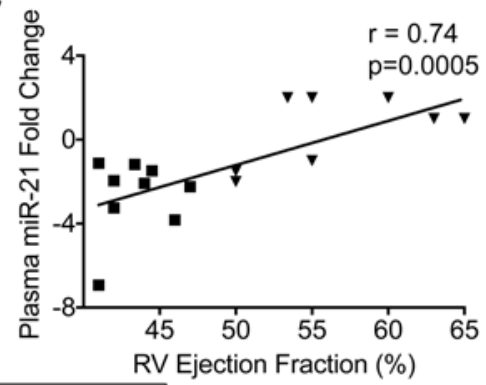

v Control

Heart Failure

D

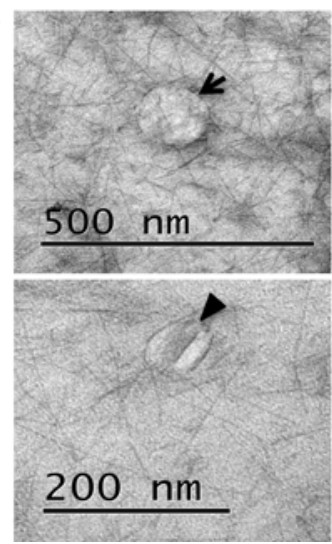

E

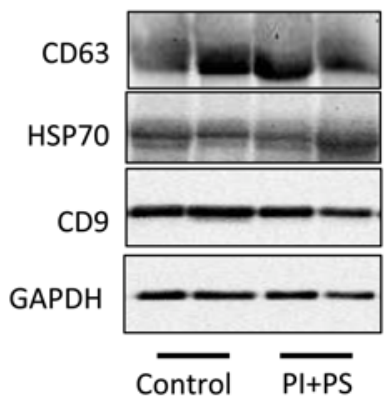

$\mathbf{F}$

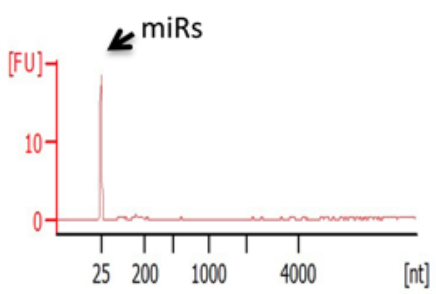

G

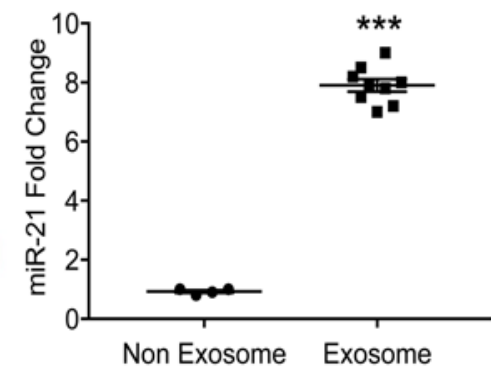

Figure 5. miR-21 is a potential biomarker for RV dysfunction in patients with tetralogy of Fallot. (A) Plasma miR-21 expression is increased in patients with $\mathrm{Pl}+\mathrm{PS}$ without RV systolic dysfunction ( $n=7)$ but switches to being decreased in patients with systolic dysfunction $(n=9)$ compared with controls $(n=12)$. (B) miR-21 expression correlates negatively with RV end-diastolic volume and (C) positively with RV ejection fraction. (D) TEM demonstrating exosomes (arrow) with characteristic cup shape (arrowhead). Scale bar: $500 \mathrm{~nm}$ (top); $200 \mathrm{~nm}$ (bottom). (E) Exosome marker expression was similar between control and PI+PS. $n=2$ /group. (F) Electropherogram of exosomes showing small RNAs in the 10- to 40-nucleotide region indicative of miRs and no contamination from larger RNAs. (G) miR-21 expression is enriched in exosomes compared with the nonexosome fraction. $n=4 /$ control; $n=9 / \mathrm{PI}+\mathrm{PS}$. RV, right ventricle; PI+PS,; TEM, transmission electron microscopy. Data are presented as mean \pm SEM. An unpaired, 2-tailed Student's $t$ test was utilized for 2-group comparisons and ANOVA with multiple testing correction for 3 or more group comparisons. Correlation was assessed using Pearson's correlation. ${ }^{*} P<0.01,{ }^{* * *} P<0.001$.

\section{Methods}

RV volume and pressure overload (PI+PS) was created in 12-week-old male FVB mice (Jackson Laboratory) (Supplemental Figure 1A). PI+PS mice were compared with age-matched sham-operated controls. Cardiac physiology was assessed using transthoracic echo, cardiac catheterization, and exercise testing performed at 1 and 2 months (compensated state with diastolic dysfunction) and at 3 months (overt heart failure, defined as the presence of edema, tachypnea, and decreased activity with systolic dysfunction). Details of surgical methods and physiologic assessment are provided in the Supplemental Methods $(16,17)$.

Histopathology. Hearts were harvested at 1,2, and 3 months, and RV and LV free wall and septum were separated and weighed. Cross sections of the hearts from PI+PS mice and sham controls were stained with Masson's trichrome for collagen deposition and TUNEL (Promega) for apoptosis $(n=6-9 /$ sham and $\mathrm{PI}+\mathrm{PS}$ for 3 time points at 1,2, and 3 months) (16). Frozen tissue sections were labeled with wheat germ agglutinin conjugate to determine myocyte cross-sectional area, and 100 cells were measured per sample. ImageJ software (NIH) was used to quantify fibrosis and cell area.

Gene and miR expression. Total RNA was isolated from the RV free wall (miRNeasy Mini Kit; Qiagen) (16). Labeled cDNA was hybridized to Agilent mouse whole genome oligonucleotide microarrays representing 41,000 probes and scanned, and probe features were extracted using Agilent feature extraction software (Agilent One-Color Microarray Low Input Quick Amp Labeling Protocol). Mice were evaluated at 3 time points after surgery; diastolic dysfunction was measured at 1 and 2 months and systolic dysfunction was measured at 3 months ( $n=6-8 /$ group/time point) and compared with sham $(n=4-5 /$ sham and PI+PS for 3 time points at 1, 2, and 3 months). qRT-PCR was used to examine expression of hypertrophy/heart failure genes, profibrotic genes, and profibrotic miRs (miR-21, miR-21*, miR-221, miR-222, and 
miR-143) at 1, 2, and 3 months $(16,18)$. For detailed methods, see the Supplemental Methods and Supplement Table 1. Microarray data have been deposited at NCBI's GEO database (accession GSE97363).

Protein expression. Protein expression of select TGF- $\beta$ pathway proteins, phosphorylated Smad2/3 (Ser245/250/255) (Cell signaling 3104), total Smad2/3 (Cell signaling 3102), PTEN (D4.3) (Cell signaling 9188), and Sprouty 1 (H-120) (Santa Cruz Biotechnology, sc-30048), was assessed by Western blot and normalized to enolase (16). Exosomal proteins CD63, HSP 70, and CD9 (SBI EXOAB-KIT-1 containing the following antibodies CD63A-1, HSP70A-1, and CD9A-1) were also assessed and normalized to GAPDH.

Plasma miR expression. Total RNA including miR was isolated from the plasma of mice with PI+PS at 1, 2, and 3 months and compared with sham controls (Qiagen) ( $n=9-10 /$ sham and PI+PS for 3 time points at 1, 2, and 3 months). C. elegans-39 (Qiagen, catalog 219610) was used as the plasma spike-in control to normalize sample loading. Plasma miR expression was assessed using a 2-step miR qRT-PCR. A Ct value $\leq 35$ cycles was considered the threshold for reliable detection of miRs, and a $\mathrm{Ct}>35$ implied that the miRs were either absent or present at very low levels $(23,24)$.

Plasma exosome expression. Total exosomes were isolated from plasma, and exosome morphology was characterized by transmission electron microscopy and purity by immunoblotting for exosomal marker proteins. RNA was isolated from the exosomes and miR expression assessed by qRT-PCR. miR expression was also assessed in the nonexosomal fraction of the plasma (52). For detailed methods, see the Supplemental Methods.

Clinical myocardial and plasma samples. Infundibular muscle bundles are routinely resected at the time of surgery if they are causing obstruction to flow of blood. RNA was isolated from RV tissue when available in children with tetralogy of Fallot undergoing initial repair and those undergoing repeat surgery for PI+PS. This was compared with children with mild RVOTO undergoing surgery and RV muscle from explanted hearts at the time of cardiac transplantation for end-stage dilated cardiomyopathy with RV failure. miR-21 expression and fibrosis was assessed as described above. RNA was also isolated from the plasma of children with PI+PS during either the compensated phase or at the time of pulmonary valve replacement and compared with controls. Controls were children with preserved cardiac function, on no cardiac medications, with no structural anomalies other than (a) incidental findings of anomalous coronary artery arising from the aorta and not the pulmonary artery or (b) those who were undergoing electrophysiology study for ablation of supraventricular tachycardia.

Statistics. Data other than microarray data sets are represented as mean \pm SEM. An unpaired, 2-tailed Student's $t$ test was utilized for 2-group comparisons and ANOVA was utilized for 3 or more group comparisons, performed on continuous, normally distributed data. $P \leq 0.05$ was considered significant for a Student's $t$ test and for ANOVA with multiple testing correction. Correlation was assessed using Pearson's correlation, with $P \leq 0.05$ being considered significant. Gene expression analysis was first performed as we have previously described, using GeneSpring GX 11.5 software, comparing PI+PS versus sham at each time point (16). Normalized data between the 20th and 100th percentiles with detected probes were used for further analysis. Quality control was performed, following which an unpaired $t$ test with Benjamini-Hochberg multiple testing correction was applied. Significantly altered gene transcripts with a corrected $P$ value of less than 0.05 and with a fold change of $\geq 2$ were considered for further analysis. Database for Annotation, Visualization and Integrated Discovery (DAVID) bioinformatics database version 6.7 was queried to identify significant KEGG pathways enriched in the above gene sets with a false detection rate of $<5 \%$.

Study approval. All procedures were performed in accordance with NIH standards and were approved by the Administrative Panel on Laboratory Animal Care at Stanford University. This study was also approved by the Stanford University Institutional Review Board, and consent was obtained for all patients, with assent obtained from guardians for children $>6$ years of age.

\section{Author contributions}

Study design, physiology testing, imaging, data acquisition and analysis, and manuscript writing were performed by SR. Experiments were conducted and data acquisition and analysis were performed by DQH. Murine surgery was performed by MZ. Murine exercise testing was performed by EB. Cardiac imaging was performed by NS. Exosome identification was performed by SGO. Protein detection was performed by GJ. Electron microscopy was performed by KBK and MC. Study design was performed by GF. Study design and manuscript writing were performed by DB. 


\section{Acknowledgments}

This work was funded by a Children's Heart Foundation grant to DB and SR; a Lucile Packard Children's Hospital Heart Center research grant to SR; a Reddy Foundation grant to SR; an American Heart Association grant-in-aid to SR; NIH grant HL061535 to DB; and the Cell Sciences Imaging Facility of Stanford University. The JEOL JEM1400 electron microscope was funded by a NIH Shared Instrumentation grant (William Talbot, PI; installation date, 01/2011). The project was supported, in part, by American Recovery and Reinvestment Act award 1S10RR026780-01 from the National Center for Research Resources (NCRR). Its contents are solely the responsibility of the authors and do not necessarily represent the official views of the NCRR or the NIH. We thank our human subjects research coordinators Sara Sherman-Levine and Aihua Zhu, supported by NIH/National Center for Advancing Translational Sciences/Clinical and Translational Science Awards grant UL1 TR001085, the Lucile Packard Foundation for Children's Health, and the Child Health Research Institute. We thank Anica Bulic for the collection of cardiac MRI data.

Address correspondence to: Sushma Reddy, 750 Welch Road, Suite 325, Palo Alto, California 94305, USA. Phone: 650.736.8752; E-mail: sureddy@stanford.edu.

1. Gilboa SM, et al. Congenital Heart Defects in the United States: Estimating the Magnitude of the Affected Population in 2010. Circulation. 2016;134(2):101-109.

2. Frigiola A, et al. Physiological and phenotypic characteristics of late survivors of tetralogy of Fallot repair who are free from pulmonary valve replacement. Circulation. 2013;128(17):1861-1868.

3. Frigiola A, Redington AN, Cullen S, Vogel M. Pulmonary regurgitation is an important determinant of right ventricular contractile dysfunction in patients with surgically repaired tetralogy of Fallot. Circulation. 2004;110(11 Suppl 1):II153-II157.

4. Rodriguez FH, et al. Outcomes of heart failure-related hospitalization in adults with congenital heart disease in the United States. Congenit Heart Dis. 2013;8(6):513-519.

5. Dinardo JA. Heart failure associated with adult congenital heart disease. Semin Cardiothorac Vasc Anesth. 2013;17(1):44-54.

6. Davlouros PA, Karatza AA, Gatzoulis MA, Shore DF. Timing and type of surgery for severe pulmonary regurgitation after repair of tetralogy of Fallot. Int J Cardiol. 2004;97 Suppl 1:91-101.

7. Lim C, et al. Early replacement of pulmonary valve after repair of tetralogy: is it really beneficial? Eur J Cardiothorac Surg. 2004;25(5):728-734.

8. Gregg D, Foster E. Pulmonary insufficiency is the nexus of late complications in tetralogy of Fallot. Curr Cardiol Rep. 2007;9(4):315-322.

9. Geva T. Tetralogy of Fallot repair: ready for a new paradigm. J Thorac Cardiovasc Surg. 2012;143(6):1305-1306.

10. Geva T. Indications for pulmonary valve replacement in repaired tetralogy of Fallot: the quest continues. Circulation. 2013;128(17):1855-1857.

11. Tardiff JC. Cardiac hypertrophy: stressing out the heart. J Clin Invest. 2006;116(6):1467-1470.

12. Rosca MG, Tandler B, Hoppel CL. Mitochondria in cardiac hypertrophy and heart failure. J Mol Cell Cardiol. 2013;55:31-41.

13. Wang Z, et al. The long noncoding RNA Chaer defines an epigenetic checkpoint in cardiac hypertrophy. Nat Med. 2016;22(10):1131-1139.

14. Ryan TD, et al. Left ventricular eccentric remodeling and matrix loss are mediated by bradykinin and precede cardiomyocyte elongation in rats with volume overload. J Am Coll Cardiol. 2007;49(7):811-821.

15. Hutchinson $\mathrm{KR}$, et al. Temporal pattern of left ventricular structural and functional remodeling following reversal of volume overload heart failure. J Appl Physiol. 2011;111(6):1778-1788.

16. Reddy S, et al. Physiologic and molecular characterization of a murine model of right ventricular volume overload. Am J Physiol Heart Circ Physiol. 2013;304(10):H1314-H1327.

17. Urashima T, et al. Molecular and physiological characterization of RV remodeling in a murine model of pulmonary stenosis. Am J Physiol Heart Circ Physiol. 2008;295(3):H1351-H1368.

18. Reddy S, et al. Dynamic microRNA expression during the transition from right ventricular hypertrophy to failure. Physiol Genomics. 2012;44(10):562-575.

19. Zhao M, Chow A, Powers J, Fajardo G, Bernstein D. Microarray analysis of gene expression after transverse aortic constriction in mice. Physiol Genomics. 2004;19(1):93-105.

20. Bang C, et al. Cardiac fibroblast-derived microRNA passenger strand-enriched exosomes mediate cardiomyocyte hypertrophy. $J$ Clin Invest. 2014;124(5):2136-2146.

21. Fichtlscherer S, Zeiher AM, Dimmeler S. Circulating microRNAs: biomarkers or mediators of cardiovascular diseases? Arterioscler Thromb Vasc Biol. 2011;31(11):2383-2390.

22. He M, et al. Plasma microRNAs as potential noninvasive biomarkers for in-stent restenosis. PLoS ONE. 2014;9(11):e112043.

23. Chen X, et al. Characterization of microRNAs in serum: a novel class of biomarkers for diagnosis of cancer and other diseases. Cell Res. 2008;18(10):997-1006.

24. Mitchell PS, et al. Circulating microRNAs as stable blood-based markers for cancer detection. Proc Natl Acad Sci USA. 2008;105(30):10513-10518.

25. Wang H, Hou L, Li A, Duan Y, Gao H, Song X. Expression of serum exosomal microRNA-21 in human hepatocellular carcinoma. Biomed Res Int. 2014;2014:864894.

26. Nollert G, Fischlein T, Bouterwek S, Böhmer C, Klinner W, Reichart B. Long-term survival in patients with repair of tetralogy of Fallot: 36-year follow-up of 490 survivors of the first year after surgical repair. J Am Coll Cardiol. 1997;30(5):1374-1383. 
27. Khairy $\mathrm{P}$, et al. Arrhythmia burden in adults with surgically repaired tetralogy of Fallot: a multi-institutional study. Circulation. 2010;122(9):868-875.

28. Oosterhof T, et al. Preoperative thresholds for pulmonary valve replacement in patients with corrected tetralogy of Fallot using cardiovascular magnetic resonance. Circulation. 2007;116(5):545-551.

29. Kozak MF, Redington A, Yoo SJ, Seed M, Greiser A, Grosse-Wortmann L. Diffuse myocardial fibrosis following tetralogy of Fallot repair: a T1 mapping cardiac magnetic resonance study. Pediatr Radiol. 2014;44(4):403-409.

30. Ashrafian H, Frenneaux MP, Opie LH. Metabolic mechanisms in heart failure. Circulation. 2007;116(4):434-448.

31. Preim U, et al. Delayed enhancement imaging in a contemporary patient cohort following correction of tetralogy of Fallot. Cardiol Young. 2015;25(7):1268-1275.

32. Wald RM, Haber I, Wald R, Valente AM, Powell AJ, Geva T. Effects of regional dysfunction and late gadolinium enhancement on global right ventricular function and exercise capacity in patients with repaired tetralogy of Fallot. Circulation. 2009;119(10):1370-1377.

33. Creemers EE, Pinto YM. Molecular mechanisms that control interstitial fibrosis in the pressure-overloaded heart. Cardiovasc Res. 2011;89(2):265-272.

34. Dickinson MG, Bartelds B, Borgdorff MA, Berger RM. The role of disturbed blood flow in the development of pulmonary arterial hypertension: lessons from preclinical animal models. Am J Physiol Lung Cell Mol Physiol. 2013;305(1):L1-14.

35. Garcia RV, VillarAV, Hurle MA, Nistal JF. Abstract 16433: TGF-beta regulates cytosolic mir-21 maturing in mouse and human cardiac fibroblasts under pressure overload. Circulation. 2013;128:A16433.

36. Cavarretta E, Condorelli G. miR-21 and cardiac fibrosis: another brick in the wall? Eur Heart J. 2015;36(32):2139-2141.

37. da Costa Martins PA, De Windt LJ. miR-21: a miRaculous Socratic paradox. Cardiovasc Res. 2010;87(3):397-400.

38. Hwang MS, et al. miR-221/222 targets adiponectin receptor 1 to promote the epithelial-to-mesenchymal transition in breast cancer. PLoS One. 2013;8(6):e66502.

39. Mendias CL, Gumucio JP, Lynch EB. Mechanical loading and TGF- $\beta$ change the expression of multiple miRNAs in tendon fibroblasts. J Appl Physiol. 2012;113(1):56-62.

40. van Rooij E, et al. A signature pattern of stress-responsive microRNAs that can evoke cardiac hypertrophy and heart failure. Proc Natl Acad Sci USA. 2006;103(48):18255-18260.

41. Patrick DM, et al. Stress-dependent cardiac remodeling occurs in the absence of microRNA-21 in mice. J Clin Invest. 2010;120(11):3912-3916.

42. Drake JI, et al. Molecular signature of a right heart failure program in chronic severe pulmonary hypertension. Am J Respir Cell Mol Biol. 2011;45(6):1239-1247.

43. Creemers EE, Tijsen AJ, Pinto YM. Circulating microRNAs: novel biomarkers and extracellular communicators in cardiovascular disease? Circ Res. 2012;110(3):483-495.

44. Pigati L, et al. Selective release of microRNA species from normal and malignant mammary epithelial cells. PLoS ONE. 2010;5(10):e13515.

45. Fijalkowska A, Torbicki A. Role of cardiac biomarkers in assessment of RV function and prognosis in chronic pulmonary hypertension. Eur Heart J. 2007;9(Suppl H):H41-H47.

46. Duong Van Huyen JP, et al. MicroRNAs as non-invasive biomarkers of heart transplant rejection. Eur Heart J. 2014;35(45):3194-3202.

47. Thum T, et al. MicroRNA-21 contributes to myocardial disease by stimulating MAP kinase signalling in fibroblasts. Nature. 2008;456(7224):980-984.

48. Arroyo JD, et al. Argonaute2 complexes carry a population of circulating microRNAs independent of vesicles in human plasma. Proc Natl Acad Sci USA. 2011;108(12):5003-5008.

49. Turchinovich A, Weiz L, Langheinz A, Burwinkel B. Characterization of extracellular circulating microRNA. Nucleic Acids Res. 2011;39(16):7223-7233.

50. Gallo A, Tandon M, Alevizos I, Illei GG. The majority of microRNAs detectable in serum and saliva is concentrated in exosomes. PLoS One. 2012;7(3):e30679.

51. Vickers KC, Palmisano BT, Shoucri BM, Shamburek RD, Remaley AT. MicroRNAs are transported in plasma and delivered to recipient cells by high-density lipoproteins. Nat Cell Biol. 2011;13(4):423-433.

52. Théry C, Amigorena S, Raposo G, Clayton A. Isolation and characterization of exosomes from cell culture supernatants and biological fluids. Curr Protoc Cell Biol. 2006; Chapter 3:Unit 3.22 